\title{
Normal heat conductivity in a strongly pinned chain of anharmonic oscillators.
}

\author{
Raphaël Lefevere ${ }^{1}$ and Alain Schenkel ${ }^{2}$ \\ 1 LPMA-UFR Mathématiques Université Paris 7, Case 7012, 75251 Paris Cedex 05, France \\ 2 Department of Mathematics P.O. Box 68 FIN-00014 University of Helsinki Finland
}

(Dated: September 19, 2018)

\begin{abstract}
We consider a chain of coupled and strongly pinned anharmonic oscillators subject to a nonequilibrium random forcing. Assuming that the stationary state is approximately Gaussian, we first derive a stationary Boltzmann equation. By localizing the involved resonances, we next invert the linearized collision operator and compute the heat conductivity. In particular, we show that the Gaussian approximation yields a finite conductivity $\kappa \sim \frac{1}{\lambda^{2} T^{2}}$, for $\lambda$ the anharmonic coupling strength.

PACS numbers: 05.70.Ln,05.60.Cd,05.20.Dd,65.40.-b,66.70.+f
\end{abstract}

The analytical derivation of the Fourier's law of heat conduction in extended Hamiltonian systems remains to this day a challenging question. See [3, 5] for a review of various aspects of the problem. A qualitative explanation for Fourier law has been provided by Peierls for the case of crystalline solids. Peierls idea essentially consists in considering the lattice excitations of the crystal, the phonons, as particles interacting weakly through the nonlinear forces acting between the atoms. By analogy with the case of dilute gases, a Boltzmann equation for phonons has been derived and refined over the years, see [8] and references therein. This approach yields a qualitative comprehension of the heat transfer in terms of collisions between phonons. The explicit computation of the heat conductivity amounts next to invert the collision operator between phonons. To this day, this computation has not been performed. This is due to the fact that the localization of the resonances in the combination of frequencies of the phonons involved in the collision is a notoriously delicate problem. In this Letter, we solve this problem in the case of a strong harmonic pinning.

Crystalline solids submitted to a heat flow are often modelized by a chain of oscillators where each oscillator move around some equilibrium position located on a regular lattice. Each oscillator interact with its nearest neigbours and also possibly with some substrate. The chain is coupled to heat baths at different temperatures by some random forcing and friction acting only on the particles at the boundaries of the chain. Although mathematical properties of such systems have been extensively studied, see 6] for a review, very little is known analytically about their physical properties, except in cases where the interactions are quadratic, 7]. Numerous numerical simulations have been performed, see [5] and references therein, strongly supporting that Fourier law is verified in pinned anharmonic chains. In particular, the conductivity of our model has been studied numerically 1, 4] and shown to be finite, although with a different scaling in temperature than what the Gaussian assumption of the Peierls theory predict, as we will see below.

As we want to concentrate on the analysis of the operator of collision between phonons, we derive a stationary Peierls-Boltzmann equation for a translation-invariant chain. The equation for the evolution of the average energy current $J_{k}$ carried by the mode of wavenumber $k$ of the chain will have the form,

$$
\frac{d}{d t} J_{k}(t)=-\gamma J_{k}(t)+\lambda \mathcal{N}_{k}(t)+S_{k}
$$

The first term on the RHS of this equation is a damping term coming from the coupling of the chain to an external friction represented by the coefficient $\gamma$. The second term comes from the Hamiltonian evolution and vanishes when the anharmonic part of the interaction is zero, i.e., when $\lambda=0$. The third term represents an external "creation" of current at constant rate. It modelizes the local temperature gradient in the non-periodic chain. Equation (11) will therefore yield a balance equation for the current carried by the mode $k$ in the stationary state. We will show that under the assumption that the stationary state is approximately Gaussian, $\mathcal{N}_{k}$ is analogous to a friction term, yielding in the end a conductivity $\kappa \sim \frac{1}{\lambda^{2} T^{2}}$. From a mathematical point of view, there is an important difference between the part coming from the external friction and the part coming from $\mathcal{N}_{k}$. Whereas the friction acts as a diagonal operator on the vector $J$, we will see that $\mathcal{N}_{k} \sim A(J)_{k}$ where $A$ is a nondiagonal linear operator. This is the collision operator of the linearized Boltzmann equation for phonons. The properties of this operator are essentially governed by resonances in the four-body interactions between phonons, which we analyze in the limit of a large harmonic pinning strength $\mu$. In particular, we show explicitly that the origin of the finite conductivity is to be found in $\mu$-dependent resonances corresponding to umklapp processes. As we concentrate only on the effect of the term coming from the nonlinearity $\mathcal{N}_{k}$, the source term $S_{k}$ is put by hand. However, in the non-translation invariant case, it comes naturally from the transport term of the equations and is roughly given by $\omega^{-1}(k) \nabla \omega(k) \nabla T$ where $\omega(k)$ is the dispersion relation and $\nabla T$ is the local temperature gradient, see [8].

The Model. We consider particles located on a periodic lattice described by their positions and momenta coordinates $\left(q_{i}, p_{i}\right)$ with $i \in 1, \ldots, N$. For the sake of notational 
simplicity, we assume that $N$ is divisible by 4 . The dynamics of the particles is governed by the Hamilton equations and an external random forcing. The Hamiltonian that we consider is of the form

$$
H(\underline{q}, \underline{p})=\sum_{i=1}^{N}\left[\frac{p_{i}^{2}}{2}+\omega^{2} \mu^{2} \frac{q_{i}^{2}}{2}+\frac{\lambda}{4} q_{i}^{4}+\frac{\omega^{2}}{2}\left(q_{i}-q_{i-1}\right)^{2}\right] .
$$

The anharmonic interaction is only in the on-site part of the Hamiltonian. The same analysis as found below may be carried out when the interaction between nearest neighbours contains also a quartic part. The fact that there is an on-site (large) harmonic interaction makes the distinction between the two cases irrelevant. We first consider the harmonic case $\lambda=0$. We introduce the Fourier coordinates for the periodic linear chain by $Q_{k}=$ $\frac{1}{\sqrt{N}} \sum_{j=1}^{N} e^{i \frac{2 \pi}{N} k j} q_{j}$, with $-N / 2+1 \leq k \leq N / 2$. The $P_{k}$ coordinates are defined in a similar fashion. We recall for further purposes that $Q_{k}^{*}=Q_{-k}$ and $P_{k}^{*}=P_{-k}$. In the complex coordinates,

$$
A_{k}^{ \pm}=\frac{1}{\sqrt{2 \omega_{k}}}\left(P_{k} \pm i \omega_{k} Q_{k}\right)
$$

with $\omega_{k}^{2}=\omega^{2}\left(\mu^{2}+4 \sin ^{2}\left(\frac{\pi k}{N}\right)\right)$, the Hamilton equations for the linear periodic chain read

$$
d A_{k}^{ \pm}= \pm i \omega_{k} A_{k}^{ \pm} d t
$$

Those equations give the temporal evolution of the amplitudes of the waves with wave number $k$ traveling through the chain in the positive or negative direction. Our (nonequilibrium) model is defined in the linear case by the system of stochastic equations

$$
d A_{k}^{ \pm}= \pm i \omega_{k} A_{k}^{ \pm} d t-\frac{\gamma}{2}\left(A_{k}^{+}+A_{k}^{-}\right) d t+d W_{k}^{ \pm}
$$

where the Wiener processes $W_{k}^{ \pm}$satisfy the relations

$d\left(W_{k}^{s} W_{k^{\prime}}^{s^{\prime}}\right) \equiv I_{k, k^{\prime}}^{s, s^{\prime}} d t \equiv 2\left[\frac{\gamma T}{\omega_{k}}+\left(s-s^{\prime}\right) \tau \alpha\left(\frac{\pi k}{N}\right)\right] \delta\left(k+k^{\prime}\right) d t$

In (5), the term in $\gamma$ is the usual friction term acting only on the momenta $P_{k}$. The Wiener processes represent the random injection of energy in the system. The first term in (6) is the usual term giving the equilibrium dynamics. In the second term of (6), $\alpha$ is an odd, dimensionless function of period $\pi$. Ultimately, the choice of physically relevant $\alpha$ is fixed by the effect of the local temperature gradient in the transport term of the non-translation invariant chain. Finally, we interpret $\tau$ in the second term of (6) as the non-equilibrium parameter of our model. When $\tau$ is zero, the periodic chain is in thermal equilibrium at temperature $T$. When $\tau \neq 0$, a stationary heat current proportional to $\tau$ is created in the chain. This is achieved, via the factor $s-s^{\prime}$, by exciting more the waves travelling in one direction than the ones travelling in the opposite direction.
In order to see those points, we recall first that the energy current in the periodic chain reads

$$
J=\frac{\omega^{2}}{N} \sum_{k=-N / 2+1}^{N / 2} \sin (2 \pi k / N) \Im\left(Q_{-k} P_{k}\right)
$$

where $\Im(z)$ denotes the imaginary part of a complex $z$. We define the two-point correlation functions in the stationary state,

$$
\Phi_{k, k^{\prime}}^{s, s^{\prime}} \equiv\left\langle A_{k}^{s} A_{k^{\prime}}^{s^{\prime}}\right\rangle
$$

where $\langle$.$\rangle denotes the average w.r.t. the stationary$ measure. Since $\Im\left(\left\langle Q_{-k} P_{k}\right\rangle\right)=\frac{1}{2}\left(\Phi_{k,-k}^{+-}-\Phi_{k,-k}^{-+}\right)=$ $\frac{1}{2}\left(\left\langle\left|A_{k}^{+}\right|^{2}\right\rangle-\left\langle\left|A_{k}^{-}\right|^{2}\right\rangle\right)$, we get

$$
\langle J\rangle=\frac{\omega^{2}}{2 N} \sum_{k=-N / 2+1}^{N / 2} \sin (2 \pi k / N)\left[\left\langle\left|A_{k}^{+}\right|^{2}\right\rangle-\left\langle\left|A_{k}^{-}\right|^{2}\right\rangle\right] .
$$

This identity expresses the fact that the energy current is the difference of the current carried by waves traveling in the positive and negative directions. From (5), we derive the equation for the two-point correlation functions,

$i\left(s \omega_{k}+s^{\prime} \omega_{k^{\prime}}\right) \Phi_{k, k^{\prime}}^{s, s^{\prime}}=\gamma \Phi_{k, k^{\prime}}^{s, s^{\prime}}+\frac{\gamma}{2}\left(\Phi_{k, k^{\prime}}^{-s, s^{\prime}}+\Phi_{k, k^{\prime}}^{s,-s^{\prime}}\right)-\frac{1}{2} I_{k, k^{\prime}}^{s, s^{\prime}}$

with $I_{k, k^{\prime}}^{s, s^{\prime}}$ given in (6). In the case where $\tau=0$, (10) is readily solved to yield, using (6),

$$
\Phi_{k, k^{\prime}}^{s, s^{\prime}}=\frac{T}{\omega_{k}} \delta\left(k+k^{\prime}\right) \delta\left(s+s^{\prime}\right) .
$$

This is of course equivalent to $\left\langle\left|P_{k}\right|^{2}\right\rangle+\omega_{k}^{2}\left\langle\left|Q_{k}\right|^{2}\right\rangle=2 T$ and $\left\langle P_{k} Q_{l}\right\rangle=0, \forall k, l$.

When $\tau \neq 0$ and for $l=-k$, one obtains from (10) and (6),

$$
J_{k} \equiv \Phi_{k,-k}^{+-}-\Phi_{k,-k}^{-+}=4 \tau \gamma^{-1} \alpha\left(\frac{\pi k}{N}\right)
$$

which is the balance equation (1) in the stationary state for the harmonic case. We note that by definition, $J_{k}=-J_{-k}$. Using (9) and the fact that $\alpha$ is an odd function, we get $\langle J\rangle=2 \omega^{2} \gamma^{-1} \tau \int_{0}^{\pi / 2} \alpha(x) \sin (2 x) d x$ for large $N$. As we interpret $\tau$ as a local non-equilibrium parameter, the conductivity in the harmonic case, $\kappa \equiv$ $\langle J\rangle / \tau \sim \gamma^{-1} \omega^{2}$, behaves as in the harmonic chain coupled to self-consistent reservoirs 22]. It reflects the fact that when $\lambda=0$ in the balance equation (1), the only term damping the current carried by each mode comes from the external friction. We will see below that the anharmonic interactions modify that behaviour of the conductivity.

Let us now consider the anharmonic case $\lambda \neq 0$. The equations of motion (5) become

$$
d A_{k}^{ \pm}=\left[ \pm i \omega_{k} A_{k}^{ \pm}-\frac{\gamma}{2}\left(A_{k}^{+}+A_{k}^{-}\right)-\frac{i \lambda}{N} R_{k}\right] d t+d W_{k}^{ \pm}
$$


where

$$
\begin{aligned}
& R_{k}=\sum_{k_{1}, k_{2}, k_{3}} \sum_{s_{1}, s_{2}, s_{3}} \delta\left(k-k_{1}-k_{2}-k_{3}\right) s_{1} s_{2} s_{3} \\
& L_{k k_{1} k_{2} k_{3}} A_{k_{1}}^{s_{1}} A_{k_{2}}^{s_{2}} A_{k_{3}}^{s_{3}},
\end{aligned}
$$

and $L_{k k_{1} k_{2} k_{3}}=\left(16 \omega_{k} \omega_{k_{1}} \omega_{k_{2}} \omega_{k_{3}}\right)^{-1 / 2}$. Here and below, unless otherwise specified, the sums are over the $k_{i}$ such that $-N / 2+1 \leq k_{i} \leq N / 2$ and $s_{i}= \pm 1$.

From (13), the equations for the $n$-point correlation functions in the stationary state,

$$
\left(\Phi^{(n)}\right)_{k_{1}, \ldots, k_{n}}^{s_{1}, \ldots, s_{n}} \equiv\left\langle A_{k_{1}}^{s_{1}} \ldots A_{k_{n}}^{s_{n}}\right\rangle
$$

read

$i \bar{\omega}^{(n)} \Phi^{(n)}=\gamma \Gamma^{(n)}\left(\Phi^{(n)}\right)+i \frac{\lambda}{N} M^{(n)}\left(\Phi^{(n+2)}\right)-\frac{1}{2} I^{(n)}\left(\Phi^{(n-2)}\right)$,

where $\bar{\omega}^{(n)}$ is the combination of frequencies,

$$
\left(\bar{\omega}^{(n)}\right)_{k_{1}, \ldots, k_{n}}^{s_{1}, \ldots, s_{n}}=\sum_{i=1}^{n} s_{i} \omega_{k_{i}}
$$

and

$$
\begin{aligned}
\left(\Gamma^{(n)}\left(\Phi^{(n)}\right)\right)_{k_{1}, \ldots, k_{n}}^{s_{1}, \ldots, s_{n}}= & \frac{n}{2}\left(\Phi^{(n)}\right)_{k_{1}, \ldots, k_{n}}^{s_{1}, \ldots, s_{n}} \\
& +\frac{1}{2} \sum_{i=1}^{n}\left(\Phi^{(n)}\right)_{k_{1}, \ldots, k_{n}}^{s_{1}, \ldots, s_{n}}
\end{aligned}
$$

$I^{(n)}$ gathers the effects of the random forcing, the special case $I^{(2)}$ being given in (6). The explicit expression of $M^{(n)}$ will be given below in the relevant cases. For $n=2$ and $k_{1}=-k_{2}=k$, one gets from (16) the balance equation in the stationary state, proceeding as to obtain (12),

$$
\gamma J_{k}+\frac{i \lambda}{N} \Psi_{k}=4 \tau \alpha\left(\frac{\pi k}{N}\right),
$$

where $J_{k}$ has been defined in (12) and

$$
\begin{aligned}
& \Psi_{k} \equiv\left(M^{(2)}\left(\Phi^{(4)}\right)\right)_{k,-k}^{+-}-\left(M^{(2)}\left(\Phi^{(4)}\right)\right)_{k,-k}^{-+} \\
&=2 i \sum_{k_{1}, k_{2}, k_{3}} \sum_{s_{1}, s_{2}, s_{3}} \delta\left(k+k_{1}+k_{2}+k_{3}\right) s_{1} s_{2} s_{3} \\
& L_{k k_{1} k_{2} k_{3}} \Im\left[\left(\Phi^{(4+)}\right)_{k, k_{1}, k_{2}, k_{3}}^{+s_{1} s_{2} s_{3}}\right],
\end{aligned}
$$

with

$$
\left(\Phi^{(n \pm)}\right)_{k_{1}, \ldots, k_{n}}^{s_{1}, \ldots, s_{n}} \equiv\left(\Phi^{(n)}\right)_{k_{1}, \ldots, k_{n}}^{s_{1}, \ldots, s_{n}} \pm\left(\Phi^{(n)}\right)_{k_{1}, \ldots, k_{n}}^{-s_{1}, \ldots,-s_{n}} .
$$

The remainder of this paper is devoted to solving equation (18) for $J_{k}$.

Closure and Linearization. In this section, we derive an explicit expression for $\Psi$ in terms of $J$. The first step is to approximate the $n$-point correlation functions (15) by products of $\Phi^{(2)}$, turning (18) into a close but nonlinear equation. This Gaussian closure assumption on the nonequilibrium stationary measure is widely used in various forms, such as in, e.g., the study of dilute gases. The second step is to linearize the resulting equation around the equilibrium solution (11). This is done in the spirit of taking a small temperature gradient or, in our case, a small parameter $\tau$.

Closing as described above the expression (19) for $\Psi$ yields zero after linearization, so that equation (18) is identical to equation (12) for the harmonic case. We thus first express $\Phi^{(4)}$ in (19) in terms of $\Phi^{(6)}$. Inverting (16) for $\Phi^{(4)}$, one can show that at lowest order in $\gamma$

$$
\Im\left[\left(\Phi^{(4+)}\right)_{k, k_{1}, k_{2}, k_{3}}^{+s_{1} s_{2} s_{3}}\right]=-\frac{\lambda}{N}\left(\Omega_{\gamma} M^{(4)}\left(\Re\left[\Phi^{(6-)}\right]\right)\right)_{k, k_{1}, k_{2}, k_{3}}^{+s_{1} s_{2} s_{3}} .
$$

where

$\left(\Omega_{\gamma}\right)_{k_{1}, k_{2}, k_{3}, k_{4}}^{s_{1} s_{2} s_{3} s_{4}}=\frac{2 \gamma}{4 \gamma^{2}+\left(s_{1} \omega_{k_{1}}+s_{2} \omega_{k_{2}}+s_{3} \omega_{k_{3}}+s_{4} \omega_{k_{4}}\right)^{2}}$,

In (21), we have dropped a term containing $\Im\left[\Phi^{(6+)}\right]$ as well as the contribution from the Itô term $I^{(4)}$, since they vanish later in the computation. We note that when $\gamma \rightarrow 0$,

$$
\left(\Omega_{\gamma}\right)_{k_{1}, k_{2}, k_{3}, k_{4}}^{s_{1} s_{2} s_{3} s_{4}} \rightarrow \delta\left(s_{1} \omega_{k_{1}}+s_{2} \omega_{k_{2}}+s_{3} \omega_{k_{3}}+s_{4} \omega_{k_{4}}\right) .
$$

We next plug (21) into (19) and use the Wick formula to express $\Phi^{(6)}$ in terms of $\Phi^{(2)}$. Linearizing around the equilibrium solution (11) results in an expression for $\Psi$ which depends solely on $J$ and contains terms which may be represented graphically by 12 topologically distinct diagrams. Those diagrams are structurally similar to the usual diagrams of the equilibrium $\lambda \phi^{4}$ theory. The main difference comes from the presence of the quantity which is the unknown of the problem, i.e., the vector $J$.

For large pinning interaction $\mu$, the result of the computation is (here and below, $c$ denotes combinatorial positive factors),

$\Psi_{k}=-\frac{c T^{2}}{\omega^{6} \mu^{6}} \frac{i \lambda}{N} \sum_{l, n}\left(\Omega_{\gamma}\right)_{k, l, n, k+l+n}^{++--}\left[J_{k}+J_{l}+J_{n}-J_{k+l+n}\right]$.

The RHS is the linear operator $A$ mentioned in the introduction, that is, the linearized collision operator of the Boltzmann equation. We remark that, in quantummechanical language, only the interactions conserving the number of phonons contribute, as the other interactions are free from resonances. We will estimate (23) in the limit of large $N$ and for small $\gamma$, more precisely, $1 \gg \gamma / \omega \gg 1 / N$. We first observe that the main contributions to the sum will arise from the resonances in $\Omega_{\gamma}$, i.e., for lattice points $(l, n)$ near the zeros of the function $f_{x}:\left[-\frac{\pi}{2}, \frac{\pi}{2}\right]^{2} \rightarrow \mathbf{R}$,

$$
\begin{aligned}
f_{x}(y, z)= & \sqrt{\mu^{2}+4 \sin ^{2}(x)}+\sqrt{\mu^{2}+4 \sin ^{2}(y)} \\
& -\sqrt{\mu^{2}+4 \sin ^{2}(z)}-\sqrt{\mu^{2}+4 \sin ^{2}(x+y+z)} .
\end{aligned}
$$


A careful analysis reveals that for $\mu^{2}>0$, the zeros of $f_{x}$ form three smooth curves. Two of these curves are obvious and given by $x+z=0$, and $y+z=0$. These resonances do not contribute to the sum, however, since the combination of $J$ 's in 23) vanishes for $k+n=0$ or $l+n=0$. This corresponds to the so-called normal processes. The third curve, corresponding to the umklapp processes, depends on $\mu$ and is difficult to localize explicitly. For large $\mu$, it is given by $x+y=\frac{\pi}{2}+\mathcal{O}\left(1 / \mu^{2}\right)$. Performing the sum over $l$ in (23) in the above-mentioned parameter regimes thus yields at lowest order in $\gamma$ and $1 / \mu($ resonances are at $l=-k+N / 2)$,

$$
\Psi_{k}=-i \lambda \frac{c T^{2}}{\omega^{7} \mu^{5}} \sum_{n} \frac{J_{k}^{-}+J_{n}^{-}}{\left|\sin \left(\frac{\pi(k+n)}{N}\right) \cos \left(\frac{\pi(k-n)}{N}\right)\right|},
$$

where we have defined $J_{k}^{-} \equiv \frac{1}{2}\left(J_{k}-J_{k+N / 2}\right)$. Before proceeding to solving equation (18), we note that any vector $J$ can be decomposed as $J=J^{+}+J^{-}$where $J^{-}$ as above and $J_{k}^{+} \equiv \frac{1}{2}\left(J_{k}+J_{k+N / 2}\right)$ have the symmetry properties $J_{k+N / 2}^{ \pm}= \pm J_{k}^{ \pm}$. It then follows from (25) that $J^{+}$does not contribute to $\Psi$, and that $\Psi=\Psi^{-}$.

Solution to the Current Equation. Recall that $J$ is odd and periodic of period $N$. We first observe that $J^{+}$does not contribute to the average current $\langle J\rangle$ given by (9). This follows from $\sum_{k} \sin (2 \pi k / N) J_{k}^{+}=0$. Since, in addition, $J$ is mapped by (25) into a vector $\Psi$ with $\Psi=\Psi^{-}$and since $J^{+}$does not contribute to $\Psi$, we need only to consider in equation (18) odd forcings $\alpha$ and odd currents $J$ satisfying $\alpha\left(\frac{\pi k}{N}+\frac{\pi}{2}\right)=-\alpha\left(\frac{\pi k}{N}\right)$ and $J=J^{-}$. We denote by $\mathcal{S}^{-}$the subspace of such vectors $J$, in which equation (18) becomes

$$
\gamma J_{k}+c \frac{\lambda^{2} T^{2}}{\omega^{7} \mu^{5}} \mathcal{L}(J)_{k}=4 \tau \alpha\left(\frac{\pi k}{N}\right),
$$

where $\mathcal{L}: \mathcal{S}^{-} \rightarrow \mathcal{S}^{-}$is given by

$$
\mathcal{L}(J)_{k}=\frac{1}{N} \sum_{k^{\prime}} \frac{J_{k}+J_{k^{\prime}}}{\left|\sin \left(\frac{\pi\left(k+k^{\prime}\right)}{N}\right) \cos \left(\frac{\pi\left(k-k^{\prime}\right)}{N}\right)\right|} .
$$

We now proceed to analyze the linear operator $\mathcal{L}$. The subspace $\mathcal{S}^{-}$has dimension $N / 4$, and a basis for $\mathcal{S}^{-}$is given by $\mathcal{J}_{k}^{n}=\sin \left(2 \pi(2 n+1) \frac{k}{N}\right), n=0, \ldots, N / 4-1$. We let $\mathcal{A}^{n} \equiv \mathcal{L}\left(\mathcal{J}^{n}\right)$. A direct computation shows that the set of $\mathcal{A}^{n}, n=0, \ldots, N / 4-1$, also forms a basis of $\mathcal{S}^{-}$ and that $\mathcal{L}$ is uniformly invertible (in $N$ ). This implies that the first term on the LHS of (26) is negligible for $\gamma$ small. Furthermore, it follows from $\sum_{k} \sin \left(\frac{2 \pi k}{N}\right) \mathcal{J}_{k}^{n}=0$ for $n \neq 0$ that only $\mathcal{J}_{k}^{0}$ contribute to the current (9). Therefore, the only contribution of the noise $\alpha$ to the current is the component of $\alpha$ along $\mathcal{A}^{0}$, say $\alpha^{0}$, where

$$
\mathcal{A}_{k}^{0} \equiv \mathcal{L}\left(\mathcal{J}^{0}\right)_{k}=2 \operatorname{sign}(k)\left(\frac{1}{4}-\left|\operatorname{sign}(k) \frac{k}{N}-\frac{1}{4}\right|\right) .
$$

One thus finally obtains from (9),

$$
\langle J\rangle \sim \frac{\omega^{9} \mu^{5}}{\lambda^{2} T^{2}} \alpha^{0} \tau,
$$

Conclusions. As mentioned in the introduction, the result of [8] shows that the local gradient of temperature imposes the choice $\alpha(k) \sim \omega^{-1}(k) \nabla \omega(k)$, that is, $\mu^{-2} \sin (2 \pi k / N)$ for large $\mu$. It has a non-zero component along $\mathcal{A}^{0}$ and the corresponding conductivity is thus given by $\kappa \equiv\langle J\rangle / \tau \sim \frac{\omega^{9} \mu^{3}}{\lambda^{2} T^{2}}$. Our explicit treatment of the resonances and the inversion of the linearized Boltzmann operator allows to justify rigorously the physical picture that the mechanism responsible for the normal conductivity in pinned anharmonic chains is the fourbody umklapp collisions between phonons. Indeed, considering a cubic instead of a quartic interaction in the Hamiltonian would yield an expression analogous to (22) but with a combination of only three frequencies. The latter is always non-zero when $\mu \neq 0$, leading to an infinite conductivity. We finally point out that when the pinning goes to zero, the localization of the resonances and the inversion of the linearized collision operator are much more difficult and there is no reason for expression (29) to be valid.

Acknowledgments. We are grateful to A. Kupiainen and J. Bricmont for numerous discussions. A.S. was supported by the Swiss National Science Foundation.
[1] K.Aoki, D.Kusnezov :Non-equilibrium Statistical Mechanics of Classical Lattice $\phi^{4}$ field theory. Annals of Physics 29550 (2002)

[2] F. Bonetto, J. L. Lebowitz, J. Lukkarinen: Fourier's law for a harmonic crystal with self-consistent stochastic reservoirs. J. Stat. Phys. 116, 783-813 (2004).

[3] F. Bonetto, J. L. Lebowitz, L. Rey-Bellet : Fourier's law: a challenge to theorists. Mathematical Physics 2000, 128151, Imp. Coll. Press, 2000.

[4] B. Hu, B. Li, H. Zhao: Heat conduction in onedimensional nonintegrable systems. Phys. Rev. E 61, 3828-3831 (2000).
[5] S. Lepri, R. Livi, A. Politi: Thermal conduction in classical low-dimensional lattices. Phys. Rep. 377, 1-80 (2003).

[6] L. Rey-Bellet: Nonequilibrium statistical mechanics of open classical systems. Proceeedings of ICMP (2003).

[7] Z. Rieder, J. L. Lebowitz, E. Lieb: Properties of a harmonic crystal in a stationary nonequilibrium state. J. Math. Phys. 8, 1073-1078 (1967).

[8] H. Spohn :The phonon Boltzmann equation, properties and link to weakly anharmonic lattice dynamics. arXiv:math-ph/0505025 